\title{
Optimization of Exit Door Locations for an Efficient Emergency Evacuation using Agent- Based Simulation and Genetic Algorithm
}

\author{
Author: Chanbin Park ${ }^{1}$ \\ Affiliation: Handong International School ${ }^{1}$ \\ E-mail: chanbinpark1015@gmail.com ${ }^{1}$ \\ <DOI:10.26821/IJSHRE.8.9.2020.8902 >
}

\begin{abstract}
Rapid urbanization exposes more people to the danger of crowd disasters. Especially, when people evacuate indoors during an emergency situation, inefficient building structures put more people in danger. Among various building elements that can be modified for faster evacuation, exit-door locations are the main variable in this paper. In order to represent the crowd movement during an emergency egress, an artificial flocking simulation, Boids, is used. Since the algorithm itself is not enough to model the complex evacuation behavior, additional manipulations in the algonthm are made. Furthermore, to optimize the exit door locations, the genetic algorithm, a randomized heuristic search strategy inspired by the theory of evolution, is redesigned for our purpose. In our modified genetic algorithm, four selection methods are presented, experimented, and compared. Ultimately, our proposed framework significantly improved the evacuation process by identifying optimal exit door locations.
\end{abstract}

Keywords: Optimization; Simulation; Genetic Algorithm; Agent-Based Model; Boids

\section{INTRODUCTION}

\subsection{Motivation}

Due to rapid growth in population and urbanization, people are becoming more exposed to the danger of crowd disasters. There are largely two types of reasons behind indoor crowd disasters. The first is overcrowding. A large number of crowds heading to the exit results in clogging, stampedes, and trampling. The second is evacuation. During emergencies, such as natural disasters(floods, earthquakes), industrial disasters(fire, chemical accidents, structural failure), or terrorist attacks, even if the number of crowds is small, people tend to move chaotic, finding a way out of the building. Safety and time are important in this situation since if people cannot evacuate fast and safely, they would be facing imminent danger. In order to achieve efficient evacuation in both cases, several building elements can be modified-emergency routes, signage systems, obstacle placement, sizes and locations of exit doors, and placing active crowd guidance [1].

Although there have been vast advances in the field of crowd behavior, it remains difficult to represent crowd behaviors in emergency evacuation [2]. Thus, this paper primarily focuses on optimizing the locations of exit doors during emergency situations through simulation, using an agent-based model(Boids), and the genetic algorithm.

\subsection{Design of the Paper}

Section 2, Crowd Model, mainly discusses the Boids algorithm and how it is manipulated to represent the movement of crowds during emergency egress. Section 3, Algorithm, introduces the simulation settings such as the design, variables, and logic of our framework. Then, it focuses on the theory and implementation of the genetic algorithm, which is used to optimize the location of exit doors. Section 4, Simulation and Results, shows how the simulation was run, as well as the results. Section 5, Conclusion, focuses on the significance of this study and points of improvement. 
Volume 8 Issue 9 September 2020

\section{CROWD MODEL}

\subsection{Introduction}

When modeling crowd behavior, there are largely three approaches: A flow-based approach, an entity-based approach, and an agent-based approach. A flow-based model considers the crowd as a whole, continuous flow of fluid. Because it focuses on the entire crowd, detailed individual behavior is not represented. An entity-based approach considers the crowd as a group of identical entities. Entities do not move by making their own decisions but move according to predefined laws. One example of an entity-based model is the Social Force Model [3], a model that represents entities as particles that move based on a set of force vectors. Lastly, there is an agent-based approach, which models individuals as autonomous agents that make decisions according to their environment. It focuses on individual attributes such as speed, emotion, etc, representing more complex behaviors [4].

Because it is important to render individual attributes of panicking people when modeling evacuation behavior, this paper took the agent-based approach. Among several agent-based modeling algorithms, this paper developed a simulation based on Boids, an artificial simulation of flocking behaviors. Normal human behaviors and flocking behaviors are very different. On the other hand, evacuating human behaviors are similar to flocking behaviors because people tend to herd during evacuation. Because of this, many pieces of research manipulated the Boids algorithm by adding more features in order to represent evacuating behavior $[5,6,7,8]$. Thus, behaviors are added to the Boids algorithm in this paper as well.

\subsection{Boids Behavior}

Boids, an artificial life simulation developed by Craig Reynolds in 1986, demonstrates coordinated animal motions such as bird flocks and fish schools. He called the moving agents "boids". The program does not control the boids as a whole but as individuals. By using three simple rules that define the movement of an individual boid, the program successfully imitates coordinated animal motion [9]. The behaviors are shown in Table 1.
Table 1. Three rules of Boids

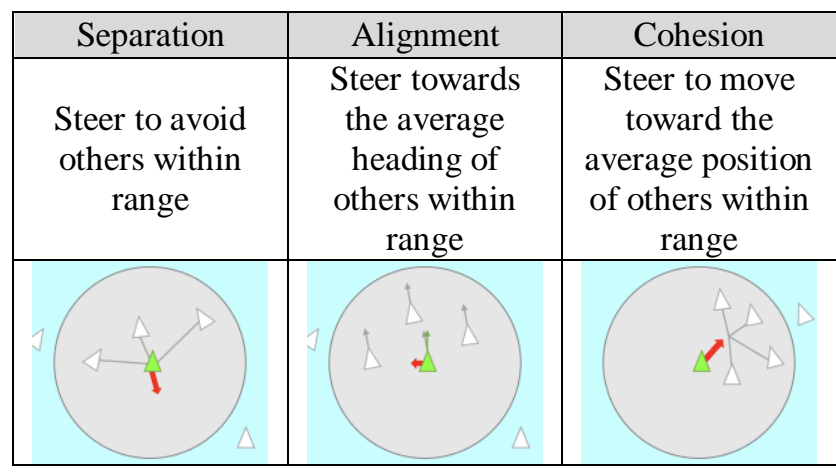

Note that the grey circle is the range that the green boid can perceive its flockmates, implemented asperceptionRadius in the next part.

Beyond these three basic rules, in order to model a further accurate model of people evacuating inside a building, two more behaviors are added, shown in Table 2.

Táble 2. Avoidance and Escape Behavior

\begin{tabular}{|c|c|}
\hline $\begin{array}{c}\text { Avoidance } \\
\text { Steer to avoid wall and } \\
\text { obstacle }\end{array}$ & Escape \\
\hline \\
\hline
\end{tabular}

The blue circle for Avoidance behavior represents the range a boid can perceive the wall and obstacle (implemented as obstacleRadiusin the next part). Whenever a block of obstacle or a wall is perceived, the green boid shoots rays (all same length) starting from the direction of its velocity and increases the angle counterclockwise until the ray does not detect a wall or obstacle. Once a ray does not detect the wall or obstacle, the boid steers toward that direction.

The yellow circle for Escape behavior represents the range a boid can detect an exit (implemented asexitRadiusin the next part). Whenever an exit is within the range, the green boid shoots a ray and checks if any obstacle touches it. If no obstacles are blocking its vision, the boid steers towards the exit. 
Volume 8 Issue 9 September 2020

\subsection{Implementation}

The following algorithms are the basic pseudocodes for the five main behaviors of a boid. Since the boids move based on the physics system, it has a position, a velocity, and an acceleration, which are all vector quantities. Also, note that the velocity vector in the pseudocode is equivalent to the heading property shown in Alignment in Table 1.

Before getting into the pseudocode, it is important to understand the concept of steering. The steering vector is equal to desired position - current position. The concept of steering would make the boids move more smoothly and thus realistically.

For the following pseudocodes, suppose $N$ number of boids are represented by, $b_{1} \ldots b_{N}$.

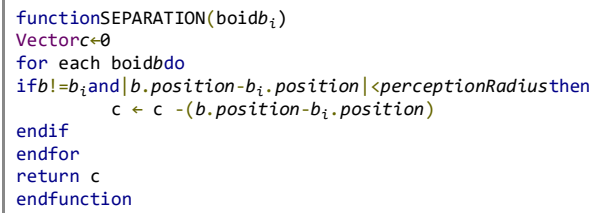

Algorithm 1. Separation Behavior

functionALIGNMENT (boid $b_{i}$ )

Vectorp $v_{i} \leftarrow \theta$

perceivedBoids $\leftarrow 0$

foreachboidbdo

b.position $-b_{i}$.position |<perceptionRadiusthen

$p v_{i} \leftarrow p v_{i}+b$. velocity

eivedBoids $\leqslant$ perceivedBoids +1

endif

$p v_{i} \leftarrow p v_{i} /$ perceivedBoids

returnpv $v_{i}-b_{i}$. velocity

end function

Algorithm 2. Alignment Behavior

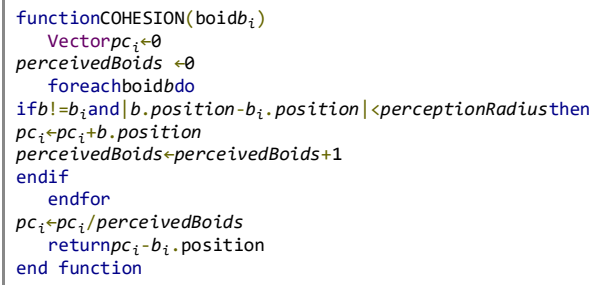

Algorithm 3. Cohesion Behavior

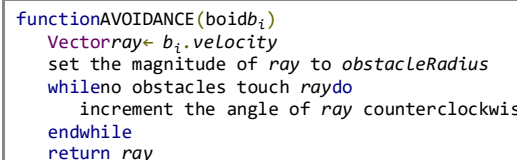

Algorithm 4. Avoidance Behavior
As shown in Algorithm 6, when a boid detects an exit, the primary vector that should affect the boid must be the vector from the function Escape. Thus, if there is an exit and no obstacles are blocking the way of the boid, the vector towards the exit is only added to the acceleration vector of the boid. If not, all the other behaviors are added to the acceleration.

The reason why the resultant vectors from Algorithm 1-5 are added to the acceleration of the boid is because Algorithm 1-5 returns a vector that represents the force. Manipulating Newton's second law $F=$ magives $a=\frac{F}{m}$. Setting all masses of boids to 1 gives $a=F$. Since acceleration is equivalent to force, return values of Algorithm 1-5 are added directly to acceleration.

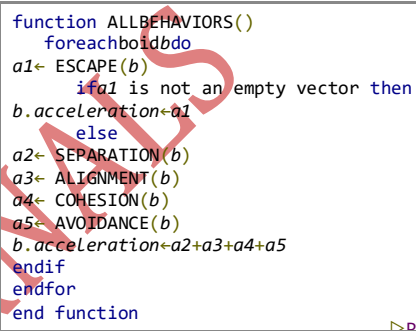

Algorithm 6. All Behaviors

\section{ALGORITHM}

\subsection{Introduction}

The genetic algorithm is a randomized heuristic search strategy or an optimization technique inspired by Charles Darwin's theory of evolution. It has been successfully applied in various optimization problems.

The algorithm first generates a randomized population of individuals (Initialization). Then, it calculates the fitness values for each candidate individual (Evaluation). Based on the fitness values, the algorithm selects two candidates and creates an offspring out of them (Selection). With a small degree of probability, the generated offspring can be mutated, or modified (Mutation). This entire process is repeated until it satisfies the termination criteria [10]. The entire process is shown as a flowchart in Figure 1.

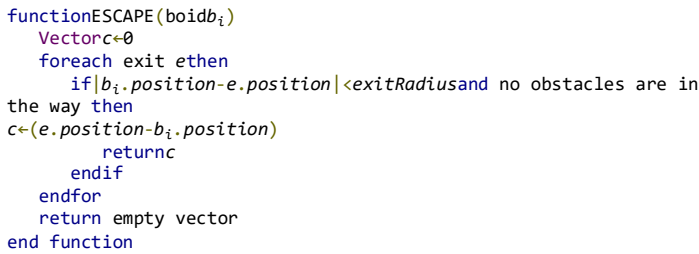

Algorithm 5. Escape Behavior 
Volume 8 Issue 9 September 2020

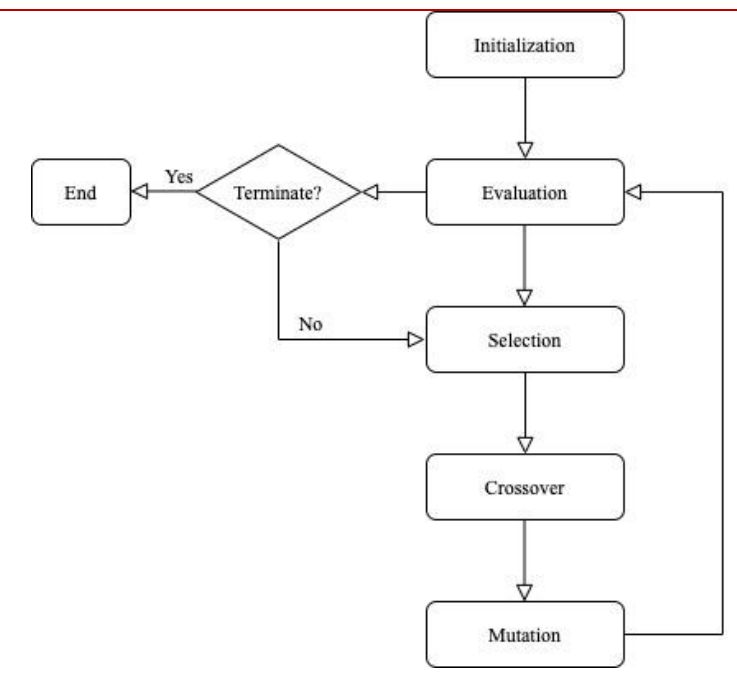

Fig 1. Genetic Algorithm Flowchart

\subsection{Setup}
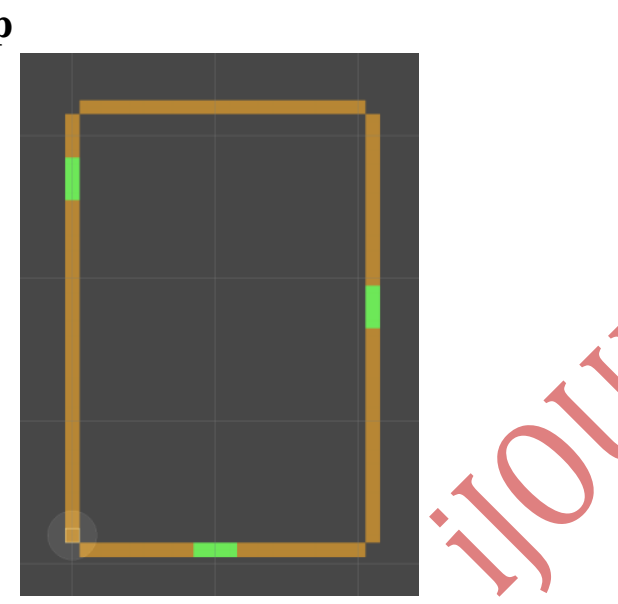

Fig 2. Cells

Cell.Let's consider Figure 2 as the environment. The outer wall of the floor is composed of small squares, which is called "cells". Each cell is considered to have a width and height of 1 . Thus, if the height is 30 , this is equivalent to 30 cells aligned vertically. A cell can either be part of a wall or part of an emergency exit. The green squares are the exit cells, and the brown squares are the wall cells. Each cell is represented as an integer. The cell in the leftmost bottom corner is 0 , and this number is incremented in a counterclockwise direction.

Figure 2 shows an environment that has a height of 30 and a width of 20. The first exit door is composed of cells of $24,25,26$, the second exit is composed of cells of $62,63,64$, and the third exit is composed of cells of $89,90,91$.

Exit Door.In Figure 3, three exit cells, 89, 90, and 91 are shown. These three exit "cells" compose one exit "door". This exit door is also represented as an integer, which is its smallest exit cell number-89 in this case.
Therefore, exit doors that have cells $(24,25,26),(62$, $63,64)$, and $(89,90,91)$, are equivalent to exit doors $24,62,89$.

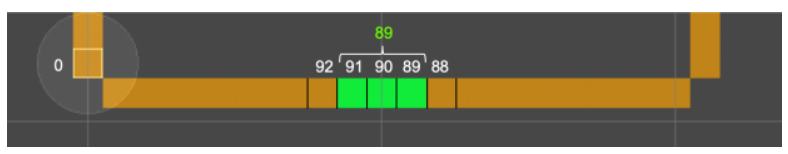

Fig 3. Exit Door Number

Genotype. Just like DNAs constitute a gene and genes constitute a chromosome, exit cells constitute an exit door and exit doors constitute a set of exit doors. A set of exit doors is defined as "genotype"(Table 3).

Table 3. GA terms applied to our system

\begin{tabular}{|c|c|}
\hline Genetic Algorithm Terms & Applied to our System \\
\hline DNA & Exit Cell \\
\hline Gene & Exit Door \\
\hline Chromosome & $\begin{array}{c}\text { Set of Exit Doors }= \\
\text { Genotype }\end{array}$ \\
\hline
\end{tabular}

System.ln order to test out each genotype, a simulation environment which contains walls, exits, obstacles, and people is needed. All of this environment would be called the "system". An example of a system is shown in Figure 4: Brown rectangles as walls and obstacles, green rectangles as exits, and white triangles as people. Each genotype is represented as green rectangles in systems. In order to do this, each system is assigned one genotype.

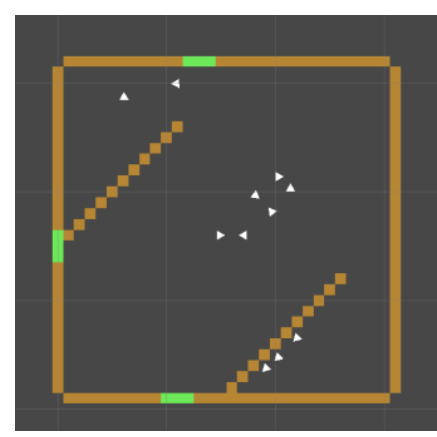

Fig 4. System

\section{Important Variables.}

$w$ : Integer. Width of the environment.

$h$ :Integer. Height of the environment.

geneSize: Integer. Number of exit cells per gene.

genotypeSize: Integer. Number of exit doors per genotype.

Genotype: Class. Contains

1.parameters: genotypeSizeamount of integers 
Volume 8 Issue 9 September 2020

\section{Fitness: fitness value}

populationSize: Integer. Number of genotypes in one generation. Also equal to the number of systems in one generation.

GenotypePopulation:GenotypeList: Contains the genotypes in the current generation.

GenotypeOffspringPopulation:Genotype List. Contains the genotypes that would be the next generation.

SystemPopulation: System List. Contains the systems in the current generation.

Functions.The functions listed in this part are essential when generating the initial population as well as doing crossover and mutation.

Figure 5 shows a gene with geneSize value of 3 . However, there can't be a gene(exit door) located at 118 or 119 , because the value of geneSize is fixed. This applies to every exit door located in the last portion of the four sides of the environment. Therefore, a gene must be at least geneSize apart from the last number of each side. A function that checks if a gene is valid is shown in Algorithm 7.

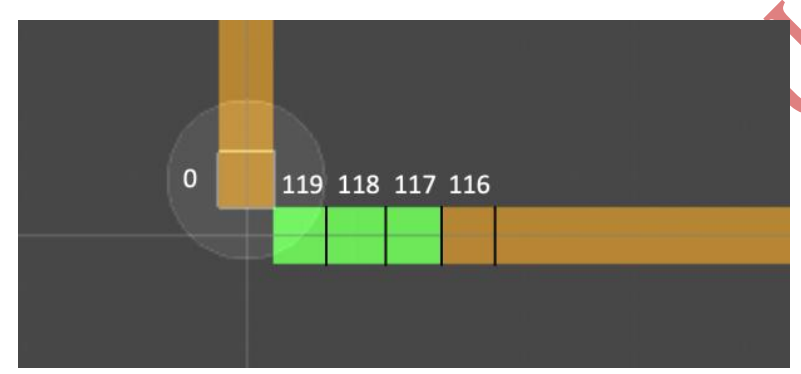

Fig 5. Exit doors located in the last part of a side

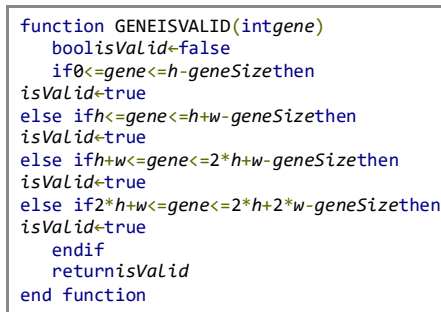

\section{Algorithm 7. Check if given gene is valid}

Moreover, when a genotypeSize number of genes are gathered to generate one genotype, no genes should overlap with each other. Algorithm 8 ensures every gene is at least one cell length apart from each other.

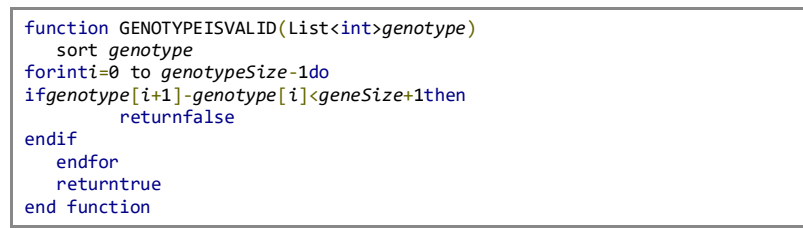

Algorithm 8. Check if given genotype is valid

\subsection{Implementation}

\subsubsection{Evaluation}

At the evaluation stage, each genotype is assigned to one system and is evaluated. Through evaluation, each genotype in GenotypePopulationgets a fitness value, and based on this value, the selection is made. Here, the value represents how much time a certain percentageEvacuationPercent of the people took to reach the exits and escape from the building.

However, since some simulation might take a long time(i.e. people do not escape for a long time), there have to be restrictions. If the system does not get a fitness value(i.e. EvacuationPercent of people did not escape) after a certain amount of time(maxEvacuationTime), the fitness value of that genotype equals maxEvacuationTime.

For our case, the smaller the fitness value, the closer it is to the answer and should more likely to be selected. However, for easier future calculations, the fitness value is modified as

$$
f_{i}=\max f-f_{i}
$$

where $\max f$ is the maximum fitness value among the genotype population. This makes the fitness value of the worst genotype as 0 and that of the best genotype highest.

\subsubsection{Selection}

There are numerous ways of selecting two genotypes from the genotype pool. In this paper, four different methods of selection are used. Before discussing the four methods, note that three genotypes with the highest fitness value are always included in the next generation(without crossover/mutation) to ensure that the performance of the algorithm is at least remaining the same, not worsening.

Complete Elitism Selection. This method randomly selects genotypes from only the three genotypes with the highest fitness values.

Roulette Wheel Selection. This method is also known as fitness proportionate selection. In this selection 
Volume 8 Issue 9 September 2020

method, the fitness value directly indicates the probability of the genotype being selected. Mathematically saying, if $f_{i}$ is the fitness value of $i^{t h}$ genotype, and $N$ is the number of genotypes, its probability $p_{i}$ to be selected is

$$
p_{i}=\frac{f_{i}}{\sum_{j=0}^{N-1} f_{j}}
$$

To implement this, first, a random float $r$ is selected, between 0 and $\sum_{j=0}^{N-1} f_{j}$. Starting from the genotype with the largest fitness value $\left(f_{0}\right)$, it must be checked if $r$ is smaller than its fitness value. If it is, select the genotype. If not, move on to the next genotype with the second largest fitness value $\left(f_{1}\right)$ and check if $r$ is smaller than $f_{0}+f_{1}$. If it is, select it. If not, move on. If the first parent is selected, do the same thing when choosing the second parent, except for selecting the first parent again.

While parent 1 and parent 2 are the indices of the genotypes that would be selected, Algorithm 9 shows the whole process of Roulette Wheel selection.

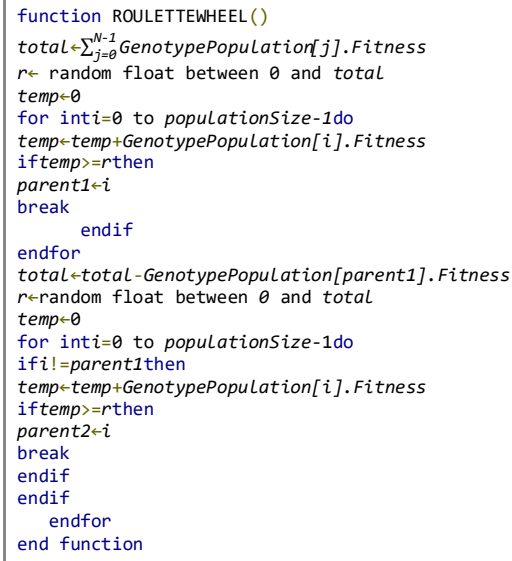

\section{Algorithm 9. Roulette Wheel Selection}

Rank Selection.This method is very similar to the Roulette Wheel selection method except that the fitness value is determined by rank, not by the fitness value itself. It "maintains a consistent selection pressure and the selection strategy is robust." [11] If $N$ is populationSize, the genotype with the highest fitness receives a rank of $N$, while the one with the lowest receives 1 . Then, the probability $p_{i}$ of a genotype to be selected is

$$
p_{i}=\frac{\operatorname{rank}(i)}{N \times(N-1)}
$$

Thus, the selection probability of a genotype with a rank of $N$ is $\frac{1}{N-1}$, while the selection probability of a genotype with a rank of 1 is $\frac{1}{N \times(N-1)}$. The other parts of this selection method are identical to the Roulette Wheel selection.

Tier Selection. In order to transmit the genes of the best genotypes as well as maintain genetic diversity, we created a selection method called Tier selection. Assume that the genotype population is sorted by fitness value in descending order. Tier selection method repeatedly selects two parents from the first three genotypes until half of the child population is filled. Then, it selects the two parents from the best half(from the first to the one that is ranked populationSize/2) of the genotype population until the child population is filled completely.

\subsubsection{Crossover}

Crossover(a.k.a. Recombination) is the process of making an offspring out of the two genotypes selected in the selection process. There are many crossover techniques, but most of themare designed for genotypes that are represented as bit arrays or strings. Table 4 is a visualization of a famous method called the single-point crossover. Through the crossover, the offspring both receive parts of genes from both of its parents.

Table 4. Single Point Crossover

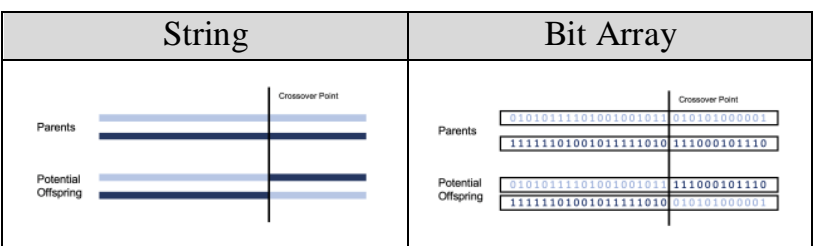

However, since the genotypes in this paper are not a string nor a bit array, the conventional method cannot be used. Thus, a new method has to be used, visualized in Table 5.

Table 5. Crossover

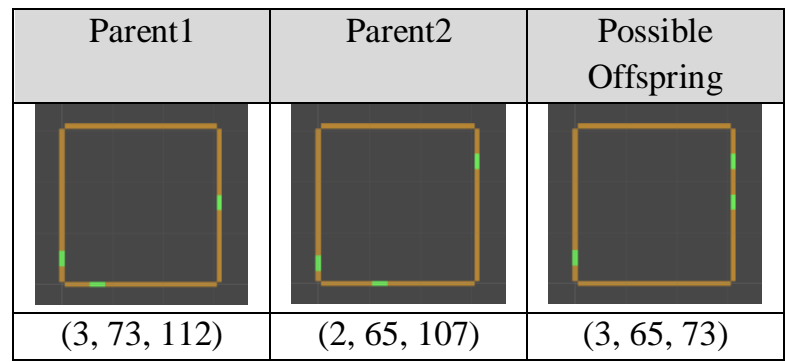

First, put every gene from the parent's genotype into a list. Then, pickgenotypeSizenumber of genes from the list and check if it is valid. The procedure is specified 
Volume 8 Issue 9 September 2020

in Algorithm 10 (GenotypeIsValid( ) was introduced in 3.2).

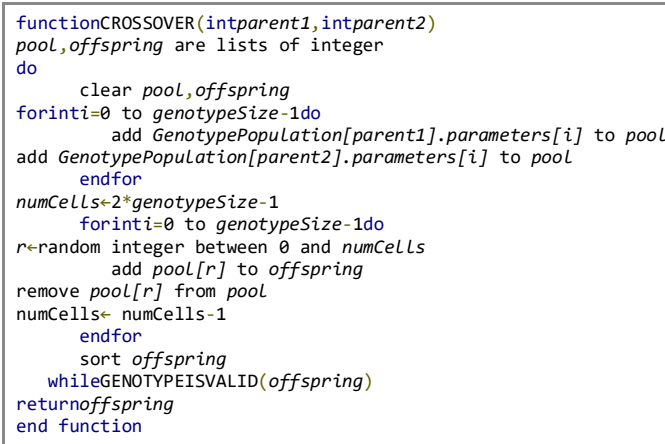

\section{Algorithm 10. Crossover}

\subsubsection{Mutation}

Mutation is a method to maintain a diverse population by mutating a gene of a genotype. If mutation is not applied, all of the offspring would be a combination of genes from the initial population. This could make the algorithm converge towards a local optimum. On the other hand, if mutation is applied too much, it would interrupt convergence. Thus, it is applied by a small degree of probability, indicated as mutationProb in Algorithm 11. In our case, mutationProb $=0.05$, or $5 \%$.

Mutation in our algorithm is equivalent to changing gene to another valid number.

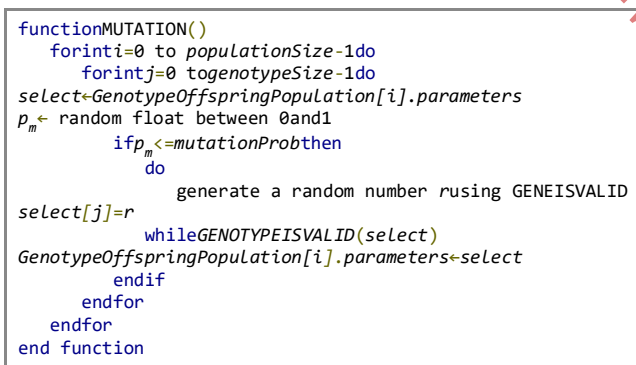

\section{Algorithm 11. Mutation}

\subsubsection{Termination}

There are a lot of ways to determine if the program should be terminated. Our program terminates once the generation number exceeds maxGenerationCount.

\section{SIMULATION AND RESULTS}

The simulation was done through Unity, a popular game engine.Initially, every system in the same generation was generated at the same time and experimented with it. However, there was time lagging between generating multiple systems, because the simulation was sequential, not parallel.Thus, the way of simulation had to be changed to experiment with one system at a time. To reduce the time, the program was run 5 times faster with Unity's built-in function. Because of this, the time it took for EvacuationPercent of the population to evacuate seems absurdly fast, but keep in mind that every evacuation time is actually 5 times slower.

Each selection method was simulated 5 times, each with a different initial population of genotypes. The same set of the initial population was applied to the four selection methods for accurate comparison. However, note that there is some discrepancy in the initial values of the results of the four methods. This happens because Unity updates the movement of boids every frame rate, which often changes. This discrepancy is trivial and does not affect the overall result.

\subsection{Simulation 1}

The first simulation was run in a simple room environment shown in Table 6.

\section{Table 6. Simulation 1}

\begin{tabular}{|l|l|}
\hline \multicolumn{1}{|c|}{ Initial Variables } & Environment \\
\hline geneSize $=3$ & \\
genotypeSize $=3$ \\
populationSize $=20$ \\
$w=30$ \\
$h=30$ \\
peopleCount= 20 \\
maxGenerationCount $=$ \\
20 \\
maxEvacuationTime $=$ \\
20 \\
EvacuationPercent $=$ \\
0.8 or $80 \%$
\end{tabular}

Table 7. Simulation 1 Results

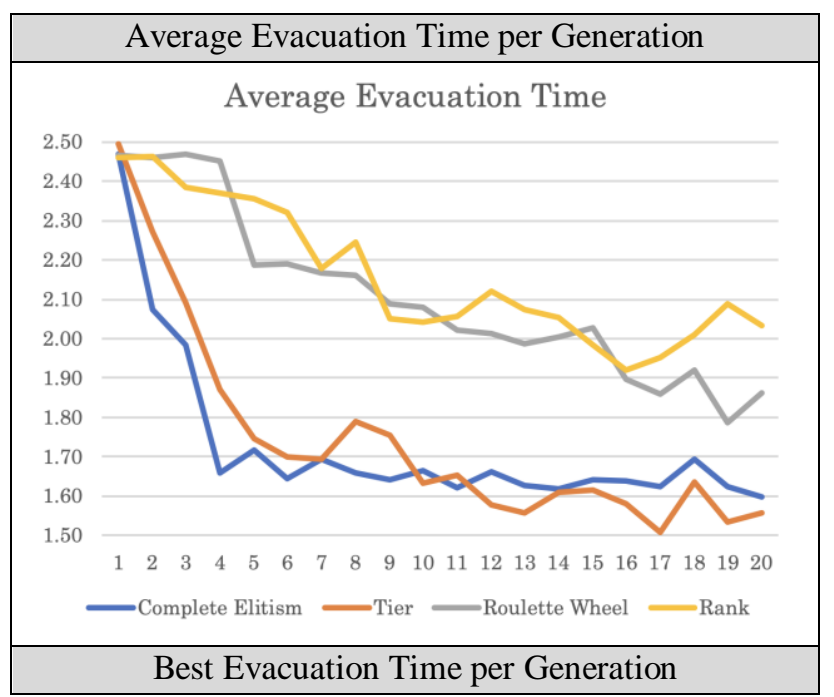


Volume 8 Issue 9 September 2020

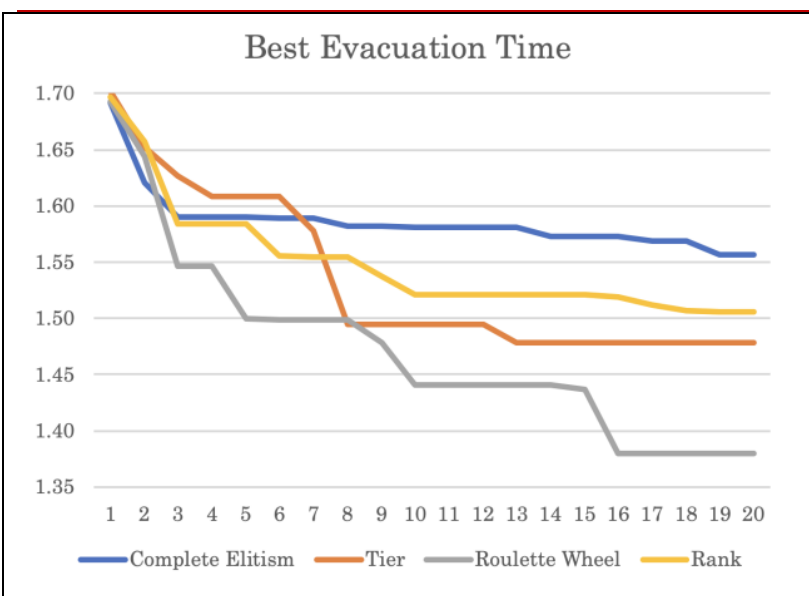

Table 7 shows the result of the simulation. The y-axis indicates the time measured in seconds for the people to evacuate, and the $\mathrm{x}$-axis indicates the generation of the genotypes. Using Tier selection method resulted in the least evacuation time for the average of the 20th generation, and Roulette Wheel selection method resulted in the least evacuation time for the best of the 20th generation.

\subsection{Simulation 2}

The second test was simulated in an environment of a coffee shop(Table 8).

Table 8. Simulation 2

\begin{tabular}{|l|c|}
\hline \multicolumn{1}{|c|}{ Initial Variables } & Environment \\
\hline geneSize $=3$ & \\
genotypeSize $=3$ & \\
populationSize $=20$ & \\
$w=38$ \\
$h=21$ \\
peopleCount $=27$ \\
maxGenerationCount \\
$=20$ \\
maxEvacuationTime $=$ \\
30 \\
EvacuationPercent $=$ \\
0.8 or $80 \%$ \\
\hline
\end{tabular}

Table 9. Simulation 2 Results

Average Evacuation Time per Generation

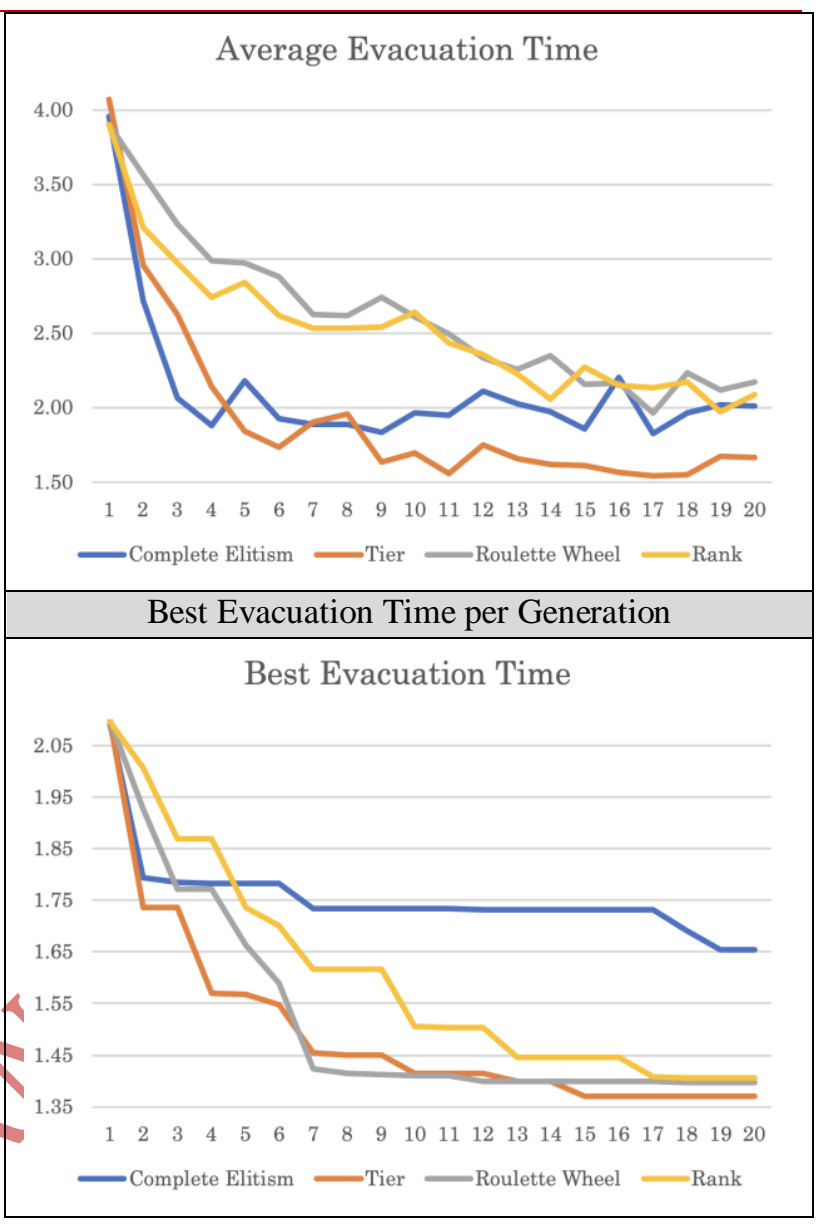

The design was based on the cafe design from http://theroomarchitects.com/cafe-1041.

Table 9 shows the result of the simulation. The y-axis indicates the time measured in seconds for the people to evacuate, and the $\mathrm{x}$-axis indicates the generation of the genotypes. Using Tier selection method resulted in the least evacuation time for both average and best of the 20th generation.

\subsection{Analysis}

Table 10 shows the performance of the four methods in simulation 1 and 2 . The selection methods are ranked based on Table 7 and Table 9. The higher the rank, the greater the fitness value, and the less time it took.

Table 10. Performance of four selection methods

\begin{tabular}{|c|c|c|c|c|}
\hline & \multicolumn{2}{|c|}{$\begin{array}{c}\text { Rank of Average } \\
\text { evacuation time of } \\
\text { last generation }\end{array}$} & \multicolumn{2}{c|}{$\begin{array}{c}\text { Rank of Best } \\
\text { evacuation time of } \\
\text { last generation }\end{array}$} \\
\cline { 2 - 5 } & Simulation 1 & Simulation 2 & Simulation 1 & Simulation 2 \\
\hline $\begin{array}{c}\text { Complete } \\
\text { Elitism }\end{array}$ & 2 & 2 & 4 & 4 \\
\hline Tier & 1 & 1 & 2 & 1 \\
\hline $\begin{array}{c}\text { Roulette } \\
\text { Wheel }\end{array}$ & 3 & 4 & 1 & 2 \\
\hline Rank & 4 & 3 & 3 & 3 \\
\hline
\end{tabular}


Volume 8 Issue 9 September 2020

First of all, the Complete Elitism selection method was second highest for average evacuation time, which is a good result, because it transfers the best genetic information to the offspring. However, since it easily falls into local optimum, the best evacuation time is ranked lowest among all four.

Secondly, the Tier selection method is ranked the highest for average evacuation time because $50 \%$ of the offspring are based on the best three genotypes, giving the best genes to the offspring, similar to the Complete Elitism selection method. Moreover, it was ranked high for best evacuation time as well because it maintains genetic diversity.

Finally, the Roulette Wheel selection method and Rank selection is ranked low for average evacuation time of the last generation because it maintains a greater genetic diversity than the two methods above. However, the Roulette Wheel selection was ranked high for the best evacuation time, while Rank selection was not. This is because Roulette Wheel selection considers the relative difference between the fitness values, while Rank selection does not and treats the differences as constant. It is reasonable to take the relative difference in fitness into account because the difference of fitness between two genotypes that are only apart by one rank can have a very large or a very small gap.

Therefore, our model proved that genetic diversity as well as considering the relative differences in fitness values are important when searching for the optimal door locations.

\section{Table 11. Best Genotypes}

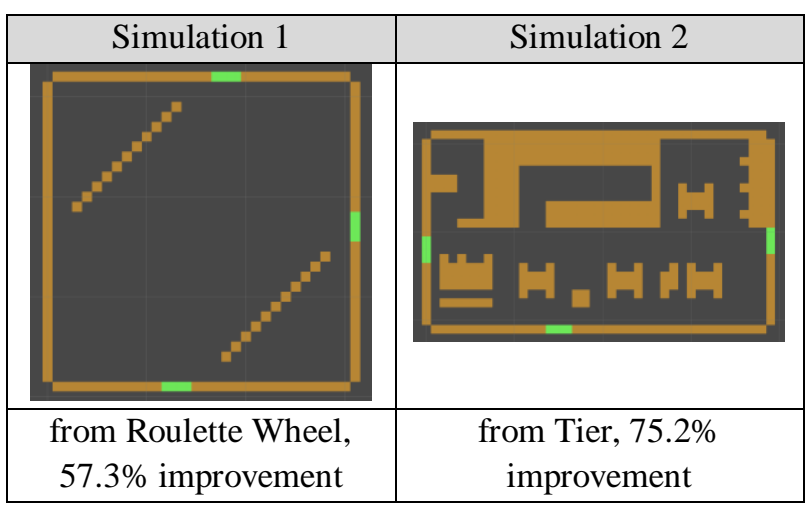

The genotypes shown in Table 11 are the best genotypes that our genetic algorithm found within 20 generations among all four selection methods. The evacuation time of the best genotype from simulation 1 showed a $57.3 \%$ of improvement, compared to the average evacuation time of its initial population( $\mathbf{1}^{\text {st }}$ generation). The evacuation time of the best genotype from simulation 2 showed a $75.2 \%$ of improvement, compared to the average evacuation time of its initial population $\left(\mathbf{1}^{\text {st }}\right.$ generation). Since no exit doors are behind the obstacles and are spread evenly, our genetic algorithm successfully worked.

\section{CONCLUSION}

Through using an agent-based simulation, Boids, with the genetic algorithm, we have provided a systematic framework to find optimal locations of a set of exit doors given an environment.

Because people tend to herd during emergency egress, many pieces of research used Boids as the basis of modeling evacuating situations. However, since the algorithm itself is not sufficient to accurately represent the complex movement of evacuating people, we added Avoidance and Escape behavior to it. Also, the genetic algorithm was redesigned. First, novel designs of genes and genotypes were created. Second, every process of the genetic algorithm-evaluation, selection, crossover, mutation, termination-were manipulated according to our gene design. Lastly, our version of Boids and genetic algorithm were simulated in Unity, a game engine, and were run multiple simulations to optimize the exit door locations during emergency egress.

Through simulating the system with four different selection methods, the algorithm showed that the average evacuation time of the last generation was minimized when experimenting with Tier and Complete Elitism selection methods. Secondly, the best evacuation time of the last generation was minimized when experimenting with Tier and Roulette Wheel selection. From this, we canconclude that a certain amount of genetic diversity as well as considering the relative differences of fitness values is important in our algorithm.

Currently, our framework only works on rectangular based environments and one floor. Thus, in the future, applying this framework to complex structures and multiple floors of buildings would be meaningful. In addition, for further accurate simulations, we would like to add more individual attributes to our boids, such as size, emotion, and more.

\section{REFERENCES}

[1]. N. Khamis, H. Selamat and F.S. Ismail et al., Optimized exit door locations for a safer emergency evacuation using crowd evacuation model and artificial bee colony optimization, 
Volume 8 Issue 9 September 2020

Chaos, Solitons \& Fractals,
https://doi.org/10.1016/j.chaos.2019.109505

[2]. Moussaïd, Mehdi et al. "Crowd behaviour during high-stress evacuations in an immersive virtual environment." Journal of the Royal Society, Interface vol. 13,122 (2016): 20160414. doi:10.1098/rsif.2016.0414

[3]. Helbing, D., \&Molnár, P. (1995). Social force model for pedestrian dynamics. Physical Review E, $\quad 51(5)$,

$4282-4286$. doi:10.1103/physreve.51.4282

[4]. Zhou, Suiping\& Chen, Dan \& Cai, Wentong\& Luo, Linbo\& Low, Malcolm Y. H. \& Tian, Feng \& Tay, Victor \& Ong, Darren \& Hamilton, Benjamin. (2010). Crowd modeling and simulation technologies. ACM Transactions on Modeling and Computer Simulation. 20. 20. 10.1145/1842722.1842725.

[5]. Zaharia, Mihai \& Leon, Florin \& Pal, Cristea\&Pagu, Gabriel. (2009). Agent-Based Simulation of Crowd Evacuation Behavior.

[6]. Chan, W. K. V., Son, Y.-J., \&Macal, C. M. (2010). Agent-based simulation tutorial simulation of emergent behavior and differences between agent-based simulation and discrete-event simulation. Proceedings of the 2010 Winter Simulation

Conference. doi:10.1109/wsc. 2010.5679168

[7]. Foudil, Cherif\& DJEDI, NourEddine. (2006). A Framework to Simulate the Evacuation of a Crowd in Emergency Situations. Georgian Electronic Scientific Journal: Computer Science and Telecommunications. Vol 1. 17-27.

[8]. S. Z. Xiuling Liu, Xiaoyu Zhu, "Modeling crowd behavior in emergency evacuation,"Advancesin information Sciences and Service Sciences(AISS), vol. 5, no. 11, 2013.

[9]. Reynolds, Craig. (1987). Flocks, Herds and Schools: A Distributed Behavioral Model. Computer $\quad 21$. 25-34. 10.1145/37402.37406.

[10]. Gandomi, Amir Hossein, et al. Metaheuristic Applications in Structures and Infrastructures. Elsevier, 2013.

[11]. Saini, Nisha. (2017). Review of Selection Methods in Genetic Algorithms. IJECS Volume 6 Issue 12 December 2017, 23261-23263. doi:10.18535/ijecs/v6i12.04 